\title{
Neonatal Rat Heart Response to Pressure Overload
}

\section{Another Difference Between the Adult and Neonatal Mammalian Heart}

\author{
Masato KAndA, ${ }^{1}$ MD and Toshio NAGAI, ${ }^{1}$ MD
}

$\mathrm{H}$ eart failure is caused by myocyte loss secondary to necrosis and/or apoptosis due to injury or excess workload, and is further complicated by adverse remodeling. Recently, there is increasing evidence refuting the traditional notion that the heart has no capacity to replace cardiomyocytes (CMs). ${ }^{1)}$ Therefore, the generation of new CMs within the cardiac milieu to replace the injured myocardium is considered one of the main therapeutic strategies for patients with heart failure and limited treatment options. The heart tissue of some teleosts and amphibians has high regenerative potential. ${ }^{2)}$ However, the innate regenerative ability of the adult mammalian heart is not adequately efficient to replace all damaged CMs; therefore, researchers are in search of the cues that induce regeneration of the myocardium.

During the embryonic and fetal periods, mammalian CMs retain growth plasticity under several environmental factors. ${ }^{3)}$ Postnatal circulatory changes (from low pressure under embryonic circulation, to high pressure under postnatal circulation) usually alter the growth pattern of the heart muscle from cell division to cell enlargement. ${ }^{4)}$ Recently, several reports have suggested that the neonatal heart regenerates CMs via $\mathrm{CM}$ proliferation after apex dissection. This suggests that significant tissue injury may be an environmental cue to enhance $\mathrm{CM}$ proliferation; however, conflicting data exist. ${ }^{5)}$ Furthermore, studies are lacking regarding the proliferation dynamics of neonatal CMs under pathological and exaggerated pressure loads.

\section{Article p.264}

On this topic, Wang and colleagues described the response of the neonatal rat heart during pressure overload. (Figure) They performed ascending aortic constriction in neonatal rats, 8-12 hours after birth. $\left.{ }^{6}\right)$ Seven days after the surgery, the hearts in the constriction group showed an increase in wall thickness without signs of CM hypertrophy. In addition, the number of CMs increased by $16.7 \%$ in the constriction group, along with an increase in proliferation and cytokinesis markers, such as Ki-67, EdU, pH3, and Aurora B-positive CMs.

The study by Wang and colleagues has several important findings. The first finding concerns their ability to overcome the technical hurdle of performing ascending aortic constriction on the $\mathrm{P} 0$ heart. ${ }^{6}$ ) Although Oliviero, et al performed ab- dominal aortic constriction on P2 rat hearts, ${ }^{7)}$ the location and the timing of the constriction in the study by Wang and colleagues are advantageous for the analysis of neonatal heart regeneration, as the cellular response to the environmental cue diverts quickly during this period. The second finding concerns the observed difference in the effect of pressure overload. In adult mice and rat hearts, pressure overload activates several pathways, including the mitogen-activated protein kinase pathway, resulting in hypertrophy and eventually heart failure via remodeling with fibrosis. ${ }^{8,9)}$ Mechanical stretch on isolated neonatal CMs, directly or indirectly (as in the case of paracrine secretion), induces hypertrophy via the same pathway that induces hypertrophy of adult CMs after constriction. ${ }^{10)}$ In this neonatal model, the hearts in the constriction group showed increased wall thickness without fibrosis, as well as improved hemodynamics and left ventricular ejection fraction via the $\mathrm{CM}$ proliferative response; however, hypertrophy was not observed. The remarkable difference between the neonatal heart, adult heart, and neonatal CMs with regard to plasticity under mechanical stress, as well as the underlying mechanisms, are intriguing, and suggest that the neonatal environment and stimulation from non-CM cells may be crucial. Further intensive studies on this topic are needed.

A previous study reported that the cell-cycle exit mechanism usually occurs during the transition from neonatal to adult heart. ${ }^{11)}$ Under pressure overload, maximum CM expansion occurred during P3-P5, and cell-cycle exit occurred during P5-P7. During mice development, many CMs undergo karyokinesis around $\mathrm{P} 0-\mathrm{P} 14$, but do not undergo cytokinesis, resulting in $80-90 \%$ binucleated cells and 10-20\% mononucleated cells. ${ }^{12)}$ In the study by Wang, et al, under pressure, a proportion of CMs completed cytokinesis, leading to proliferation. During the process, dedifferentiation was observed, including the disassembly of the sarcomere structure. ${ }^{6}$ Several cytokinesis stimulators, such as the Neuregulin 1/Erb B2 pathway, or restrictors, such as Hippo signaling, have been reported. ${ }^{1)}$ Further studies are required to clarify, at the cellular and molecular levels, whether the aforementioned mechanisms occur during pressure overload, or if a novel mechanism exists in this model.

The other unanswered question in this study concerns the relationship between non-CMs and the increase in CMs. ${ }^{6}$ The

From the ${ }^{1}$ Department of Cardiovascular Medicine, Chiba University Graduate School of Medicine, Chiba, Japan.

Address for correspondence: Masato Kanda, MD, Department of Cardiovascular Medicine, Chiba University Graduate School of Medicine, 1-8-1, Inohana, Chuo-ku Chiba, Chiba 260-8677, Japan. E-mail: mkanda5315@yahoo.co.jp

Received for publication January 4, 2017. Revised and accepted January 30, 2017.

Released in advance online on J-STAGE March 27, 2017.

All rights reserved by the International Heart Journal Association. 

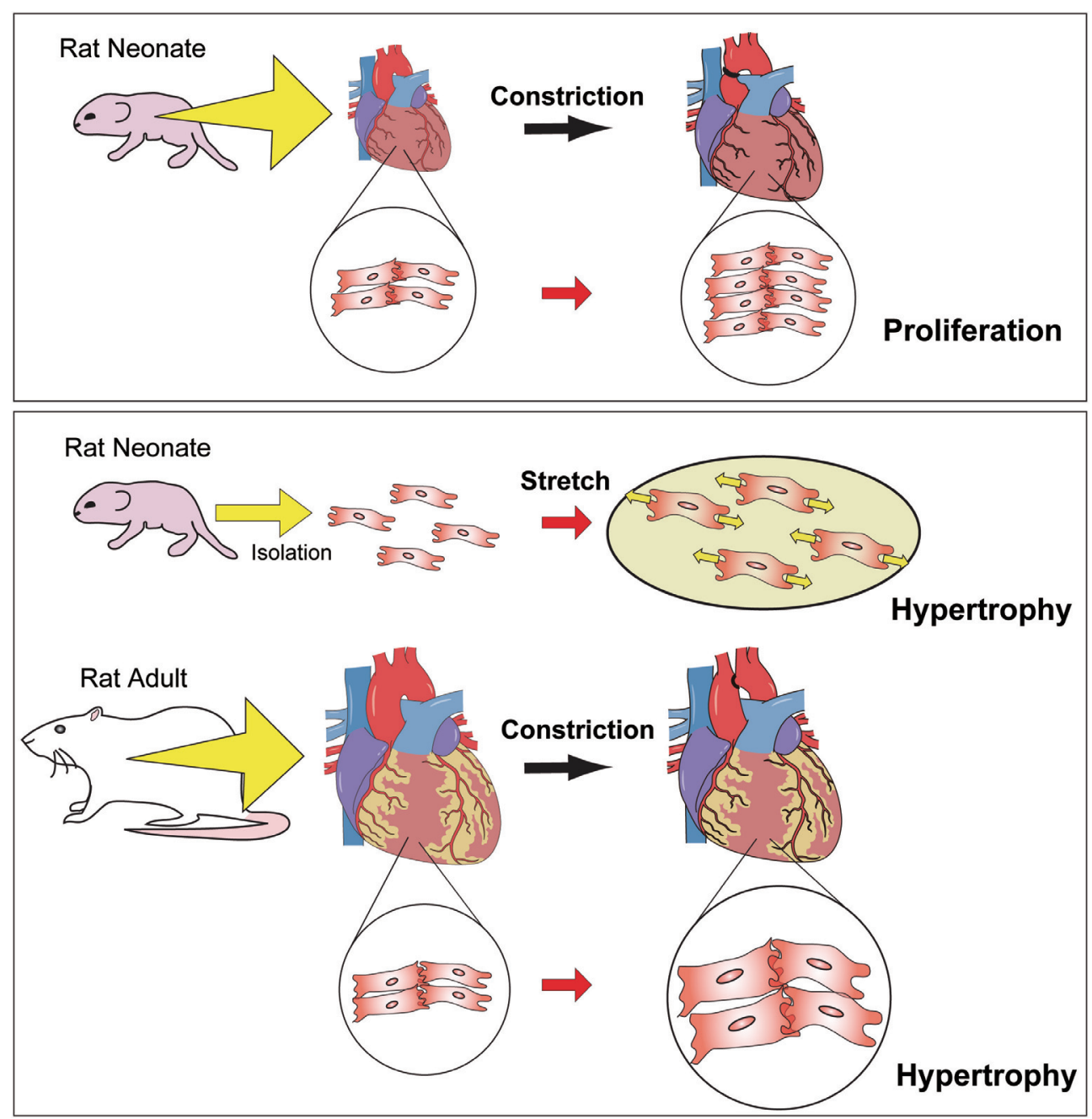

Figure. Difference between adult and neonate rat cardiomyocyte response towards pressure overload. Adult cardiomyocytes under transverse aortic constriction or neonate isolated cardiomyocytes under mechanical stretch in vitro showed hypertrophic change. In contrast, neonate cardiomyocytes under ascending aortic constriction showed proliferative response. Note that in the neonate operation performed by Wang, et al, constrictive pressure increases as the size of the aorta increases during P0-P7.

$16.7 \%$ increase in CMs does not completely account for the $50 \%$ increase in left ventricular wall thickness. The increase in non-CMs, such as cardiac fibroblasts or mesenchymal cells, may contribute to the volume increase directly or via an increase in the extracellular matrix which contain the cells. ${ }^{13)}$ In addition, stimulated non-CMs may indirectly intervene with $\mathrm{CM}$ proliferation. Ieda, et al reported that embryonic cardiac fibroblasts induce CM proliferation via fibroblast-specific signals whereas adult cardiac fibroblasts promote CM hypertrophy. ${ }^{14)}$ Finally, other mechanisms, such as the activation of dormant cardiac progenitor or stem cells, may contribute to the high tolerance for pressure overload with regard to cardiac growth and function. Performing a genetic lineage trace in this pressure overload model may provide answers to this question.

Overall, this study describes the regenerative potential of neonatal rat hearts, and an analysis of the underlying mechanisms may shed light on the search for cardiac regeneration stimulators in mammals.

\section{REFERENCES}

1. Foglia MJ, Poss KD. Building and re-building the heart by cardiomyocyte proliferation. Development 2016; 143: 729-40. (Review)

2. Poss KD. Advances in understanding tissue regenerative capacity and mechanisms in animals. Nat Rev Genet 2010; 11: 710-22. (Review)

3. Porrello ER, Widdop RE, Delbridge LM. Early origins of cardiac hypertrophy: does cardiomyocyte attrition programme for pathological 'catch-up' growth of the heart? Clin Exp Pharmacol Physiol 2008; 35: 1358-64. (Review)

4. Klopfenstein HS, Rudolph AM. Postnatal changes in the circulation and responses to volume loading in sheep. Circ Res 1978; 42: $839-45$.

5. Aurora AB, Porrello ER, Tan $\mathrm{W}$, et al. Macrophages are required for neonatal heart regeneration. J Clin Invest 2014; 124: 1382-92.

6. Wang Z, Schmull S, Zheng H, Shan J, Zou R, Xue S. Ascending aortic constriction promotes cardiomyocyte proliferation in neonatal rats. Int Heart J 2017; 58: 264-70.

7. Oliviéro P, Chassagne C, Kolar F, et al. Effect of pressure overload on angiotensin receptor expression in the rat heart during early 
postnatal life. J Mol Cell Cardiol 2000; 32: 1631-45.

8. Yuan Y, Zong J, Zhou H, et al. Puerarin attenuates pressure overload-induced cardiac hypertrophy. J Cardiol 2014; 63: 73-81.

9. Boluyt MO, Robinson KG, Meredith AL, et al. Heart failure after long-term supravalvular aortic constriction in rats. Am J Hypertens 2005; 18: 202-12.

10. Shyu KG. Cellular and molecular effects of mechanical stretch on vascular cells and cardiac myocytes. Clin Sci (Lond) 2009; 116 377-89. (Review)

11. Ahuja P, Sdek P, MacLellan WR. Cardiac myocyte cell cycle control in development, disease, and regeneration. Physiol Rev 2007;
87: 521-44. (Review)

12. Takeuchi T. Regulation of cardiomyocyte proliferation during development and regeneration. Dev Growth Differ 2014; 56: 402-9. (Review)

13. Schmuck EG, Mulligan JD, Ertel RL, et al. Cardiac fibroblast-derived $3 \mathrm{D}$ extracellular matrix seeded with mesenchymal stem cells as a novel device to transfer cells to the ischemic myocardium. Cardiovasc Eng Technol 2014; 5: 119-31.

14. Ieda M, Tsuchihashi T, Ivey KN, et al. Cardiac fibroblasts regulate myocardial proliferation through betal integrin signaling. Dev Cell 2009; 16: 233-44. 